\title{
EVALUATION OF SOCIETAL IMPACT IN NORWEGIAN SSH EVALUATIONS
}

\author{
JON HOLM AND ANETTE ASKEDAL
}

DOI: $10.22163 /$ fteval.2019.382

\section{INTRODUCTION}

$\mathrm{I}$ n this paper we present how evaluation of societal impact of research was introduced in national research evaluations in Norway within social sciences and the humanities through an adaptation of the "Research Excellence Framework" (REF) 2014 impact case method. We focus on the practical aspects of this introduction, the processes of evaluation and the impact of the impact evaluation on the discourse on societal benefits of "Social Sciences and Humanities" (SSH) research. Finally, we discuss the limitations of the impact case method and indicate some possible ways forward.

\section{THE INCLUSION OF SOCIETAL IMPACT}

The Research Council of Norway (RCN) has been performing nationwide research evaluations for over 20 years. The interval of these evaluations is approximately 10 years which means that nearly all academic subjects have now been evaluated twice. The aim of the subject-specific evaluations is to provide a critical review of the Norwegian research system in an international perspective, and to provide recommendations on measures to encourage increased quality and efficiency of research. The evaluations help to ensure that the RCN has the necessary information on which to base its strategic research funding and efforts vis-à-vis public bodies. The evaluations also serve as a tool for the institutions themselves in their ongoing efforts to refine their own strategic and scientific framework. ${ }^{1}$ There is no direct link to funding.

Traditionally, the national research evaluations have focused on the quality and efficiency of research activities at the national, institutional and group level. As a response to the political expectations of harvesting societal benefit from increased investments in research, the RCN decided to include societal impact as a dimension of the latest evaluations of the humanities (2017) and social sciences (2018). The large majority of researchers in Norway within the relevant disciplines were included in the two evaluations.

The main method used to assess societal impact in the two evaluations was borrowed from the "2014 Research Excellence Framework" in the UK. The method was chosen for two main reasons: 1) It was well documented, tested and evaluated2, and 2) the definition of impact used in the REF was judged to be sufficiently broad to include most of the expected societal benefits from SSH research: "an effect on, change or benefit to the economy, society, culture, public policy or services, health, the environment or quality of life, beyond academia"s

In contrast to more traditional methods for measuring societal and economic benefit, like counting patents or spin-off companies, we saw the REF definition as more open to disciplinary differences and compatible with the multitude of pathways to impact documented in empirical studies. ${ }^{4}$

\section{INTRODUCING SOCIETAL IMPACT TO THE SSH INSTITUTIONS}

Choosing an existing method to assess societal impact made the task of introducing a new evaluation dimension to the national evaluation system in Norway more manageable. The main effort of the RCN then went into convincing the Norwegian higher education institutions that the REF impact case template could actually be used to document the societal benefits resulting from SSH research in a meaningful way.

When planning the evaluation of humanities research in 2013, the international debate on the public value of the humanities was making its waves felt also in Norway. There was a strong resistance in academia against thinking of humanities research in terms if usefulness. At the same time, proclamations on the essential role of the humanities for the development of society were manifold. In other words, there was a discrepancy between the feeling of importance in academia and the ability to document how research results had been put into use and to point out the actual beneficiaries.

The impact case method also received various types of criticism from the researcher community. The most common objections were that the cases only covered a small part of the societal relevance of an institution, they implied a linear relationship between research and impact, they were not reflecting the complexities of researcher - user relations and not covering the important impacts taking place within academia.

With this in mind, the RCN invited representatives from institutions that took part of the evaluation to an impact-workshop. The aim of the workshop was to explore how the institutions could use the REF impact case template to describe the pathways from research to societal impact according to the REF definition. The participants were introduced to the REF case-model by Professor Helen Small - a literary scholar and from Cambridge University - who had had a leading role in her faculty's impact case submissions to the REF. 
During the workshop, many participants took the opportunity to discuss how they could use the REF impact template to describe specific societal benefits from research at their institutions. In this way, the workshop produced a change in the discourse from an essentialist question of what impact is to a pragmatic question on how to document the societal benefits of research. This change in attitude was crucial for the success of the evaluation exercise. There is a fundamental difference between the effort of understanding and conceptualising a certain phenomenon like the societal benefit from research to the task of actually establishing a new practice of documenting societal impact. The debate on how to document and assess the societal benefits from research should thus not be limited to a discussion of the meaning of a certain concept or theory on the role of science in society. In order to inform policy, the debate should also take into account how political expectations for societal benefits from investments in research are implemented through evaluation exercises or regulatory regimes.

An interesting example of this implementation perspective is given by Marta Natalia Wróblewska, who in a recent PhD-theses investigates the process of constructing the notion of impact in the British REF. Inspired by Michel Foucauld's theory of governmentality, Wróblewska (2018) argues that the resulting understanding and practices of societal impact "is a response to a set of struggles over issues related to selecting a new direction for the economic development (knowledge-based economy), reshaping the role of universities in society /third mission, entrepreneurial university), as well as class issues and tensions between particular aca-

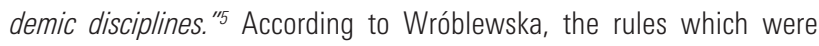
introduced with the REF guidelines could be considered as empty and unfinalised before they were taken into use and translated into concrete practices at the research institutions and thus forming an impact infrastructure consisting of professional roles, teaching frameworks and specified procedures and timeframes.

\section{THE EVALUATION PROCESS}

The RCN collected a total of 404 impact cases from the participating institutions and research groups for the evaluations of humanities and social sciences (170 cases were submitted to humanities evaluation and 234 cases to social sciences evaluation). The submission of impact cases was optional and for that reason the number of impact case per researcher varied a lot among institutions with an average of one case per 13 researchers.

For both evaluations the RCN carried out a brief descriptive analysis of the categories of impact that was reported in the impact cases. The purposes of these analyses were not to evaluate the cases, but to describe trends in the submitted material. The analysis showed that research leading up to the reported impact was commonly conducted in groups, that the geographical reach was national, and that the most common channel from research to impact was user-oriented dissemination. For the social science cases, the most common beneficiary of the impact were political institutions, and the principal registered effect was political. The general public was the most common beneficiaries for the cases within humanities, and the principal effect registered was cultural.

The RCN also did a mapping of the impact cases onto the thematic priorities within Horizon 2020 (H2020) societal challenges and those indicated by the Norwegian government's long-term plan for research and higher education. The somewhat surprising result was that there was a greater match with the European priorities than with the Norwegian priorities. This was to a great extent due to the presence to the $\mathrm{SSH}$-related theme "Europe in a changing world" in H2O2O.

The evaluation of the impact cases was carried out by the same international peers who evaluated the quality of Norwegian research. The evaluation panels found several good and varied examples of societal impact among the submitted cases. In the humanities evaluation the committee was "favourably impressed with the range and depth of societal impacts from the Humanities", and in the evaluation of social sciences the evaluators found that the research had "considerable relevance to a large range of public and private societal actors and activities" . The evaluators highlighted 64 cases as examples of good practice. These were cases that documented concrete and significant proof of impact on society.

\section{CHALLENGES}

Despite this, the evaluators experienced a number of difficulties when trying to assess societal impact in the two evaluations, and the evaluation task was described as "particularly challenging"8 in the evaluation of social sciences. The evaluators found that there was an uneven understanding of the meaning of impact among the participating institutions and research groups. A majority of the submitted impact cases merely described communication activities, rather than providing documentation of societal impact. For this reason, the panels found it difficult to assess several of the submitted impact cases, and they recommended that the institutions developed a more strategic approach to impact, and also that the difference between impact and engagement was better defined for the institutions. In addition, the evaluators recognised that there were many methodological difficulties linked to the assessment of societal impact, and they saw a need for further development of the methods for assessing and evaluating societal impact, and also for more sophisticated tools for gathering and articulating evidence of impact.

The RCN has used impact case descriptions as the main source for evaluating societal impact also in other recent evaluations (including evaluations of research institutes and thematic evaluations). The reported difficulties have been the same in most of these evaluations. In many cases the distinction between societal impact and dissemination is not clear. We take this as an indication that researchers and institutions have not fully understood the expectations embedded in the REF impact case genre. The different interpretation of impact, and also the failure to document actual change, made it difficult for the experts to assess a number of cases.

The RCN recognises, in order to make robust assessments of the societal impact of research, that there is a need to combine different methods. For that reason user-surveys and interviews were included in some of RCN's recent evaluations in order to add a users' perspective to the assessment of societal impact. It was however problematic to use the result of the surveys in most of the evaluations. The response rate was sometimes very low, and the internal response rate varied between the different sets of questions. As a result, the evaluators placed more emphasis on impact cases than on survey results when assessing the societal impact of an institution. 


\section{THE IMPACT OF THE IMPACT EXERCISE}

Despite the many methodical challenges in impact evaluations, the RCN has received positive feedback from the institutions and researchers on the usefulness of the impact-exercise. Several of the impact cases produced for the evaluations have been used by the institutions and researchers themselves e.g. published on the institutions websites or included in the researcher's CV. We also see signs of a more systematic approach in the institutions in identifying and documenting the (potential) societal impact of research.

The impact case method has also given valuable new knowledge in the variety of ways in which SSH research creates societal benefits. We have thus moved from a situation with a rather vague discourse on SSH-research as a general societal good to a collection of concrete evidence that could be used in a debate on how research funding should be attributed in order to obtain specific societal (or commercial) aims. As an example, the impact cases from the humanities were used in policyadvice to the government related to the white paper on the humanities that was launched during the evaluation. ${ }^{9}$ It is however important to note that a collection of 404 impact cases cannot give a representative picture of the societal impact of SSH research in Norway.

\section{THE WAY FORWARD}

So, where do we go from here? There is a rising demand from policymakers and funders that potential societal benefit should be considered through the whole life cycle of the research process onto the application of results. In this perspective, the difficulties reported by the evaluation committees in assessing the actual impact of Norwegian SSH research is a cause of concern. Based on our experience with the recent evaluations in the RCN we would argue that there are two aspects that needs to be addressed in the time to come:

- further development of the impact infrastructure at the institutions,

- further development of the methods for assessing and evaluating societal impact;

\section{FURTHER DEVELOPMENT OF THE IMPACT INFRASTRUCTURE:}

The evaluation committees' recommendation to the institutions to take a more strategic approach to impact documentation is in our view a result of an underdeveloped impact infrastructure at the institutions. This is not only a problem for policy-makers and funders searching for a return on their investments in research. It is also a problem for the academic institutions themselves that are confronted with an expectation to document societal benefits from their research, but lacking the impact infrastructure that will help them to identify, document and learn from how research produced at their institutions in the past have led to positive (or negative) effects in society.

\section{FURTHER DEVELOPMENT OF THE IMPACT METHODOLOGY:}

The evaluation committees also calls for further development of the methods for assessing and evaluating societal impact, and for more sophisticated tools for gathering and articulating evidence of impact. As earlier noted, the RCN recognises, in order to make robust assessments of the societal impact of research, that there is a need to combine different methods. In addition to this, it might also be useful to change the focus. In a recent report by two Norwegian evaluation experts on the concept and practice of societal impact ${ }^{10}$, it is argued that the object of evaluation should shift from the research results and their dissemination towards the process of interaction between researchers and users. They also argue that the evaluation of impact needs to be related to the actual goals of the research performing institutions.

The RCN is currently investigating the possibility of creating a national evaluation protocol in Norway that will allow the higher education institutions to take a larger responsibility for the evaluation of their own activities as it is done under the Dutch "Standard Evaluation Protocol".1" Our hypothesis is that evaluation results will be more relevant for the strategic development of each institution if the evaluation criteria are aligned with their strategic goal. Giving the higher education institutions a greater responsibility for the evaluation of their own activities, will probably also tie the evaluation processes more closely to the research processes, creating loops of feedback from evaluation results to the managers of research projects, groups and departments.

\section{CONCLUSION}

One of the main lessons of the recent evaluations of SSH in Norway is how a pragmatic approach to assessing societal impact contributed to a change in the way that academics and institutional leaders talk about the societal benefits from research in Norway. Although better definitions and conceptualisations of evaluation criteria - such as societal impact - are always welcome, our experience is that the evaluation process in itself created a new understanding of the phenomenon to be evaluated.

Recommendations provided by evaluation experts based on the recent evaluations in Norway and cases of international best practice, could indicate that future evaluation exercises in Norway - including societal impact - should be more closely linked to the purposes and strategic goals of the research organisations in order to allow these organisations to experiment with different kinds of evaluations methods and processes that are more in tune with the actual research processes and the multitude of ways that researchers interact with partners outside of academia.

So far, the national research evaluations in Norway have served an important function in the implementation of national policies for research and higher education. The impact of the latest evaluations in SSH - changing the way that societal impact of SSH research is conceived and discussed - is an example of this transformative role. In the choice of future model for research assessment in Norway, there is thus a balance to be struck between the need for a better adaptation of evaluation criteria to the strategic goals of each institution and the use of research evaluations as a policy instrument at the national level. It remains to be seen if it will be possible to move the evaluation processes and stewardship closer to the research institutions, while assuring at the same time that such institutional evaluations respond to national policy needs. 


\section{REFERENCES}

Manville, C., Guthrie, S., Henham, M-L., Garrod, B., Sousa, S., Kirtley, A., Castle-Clarke, S. and Ling, T. (2015). Assessing impact submissions for REF 2014: An evaluation. RAND Corporation, Santa Monica, Calif. And Cambridge, UK.

Gulbrandsen, M. and Sivertsen, G. (2018). Impact i anvendt forskning: begrepsavklaring og praksis. NIFU Report [Impact in applied research concepts and practice. Forthcomming]

Ministry of Education and Research (2017). Humaniora i Norge [The Humanities in Norway] (Meld. St. 20 2016-2017)). Oslo.

REF2014 Reserach Excellence Framework (2011). Assessment framework and guidance on submissions, Bristol

The Research Council of Norway (2017). Evaluation of the Humanities in Norway, Oslo.

The Research Council of Norway (2018). Evaluation of the Social Sciences in Norway, Oslo.

Thune, T. et al. (2014). Noder i kunnskapsnettverket: Forskning, kunnskapsoverføring og eksternt samarbeid blant vitenskapelig ansatte i UHsektoren. [Internal and external cooperation patterns among academic staff in the Higher Education sector in Norway] NIFU Report 2014:23. Oslo.

VSNU, KNAW and NWO (2014). Standard Evaluation Protocol 2015 2021, Voorburg.

Wróblewska, M. N. (2018). The making of the Impact Agenda: A study in discourse and governmentality governmentality (PhD Thesis Synopsis, p.7). Warwick University, Coventry, United Kingdom, Retrieved from: https://tinyurl.com/y2nkhlwe 25 February 2019

\section{AUTHORS}

\section{JON HOLM}

The Research Council of Norway

Drammensveien 288, 0283 0slo (Norway)

E: jon.holm@rcn.com

\section{ANETTE ASKEDAL}

The Research Council of Norway

Drammensveien 288, 0283 Oslo (Norway)

E: anette.askedal@rcn.com

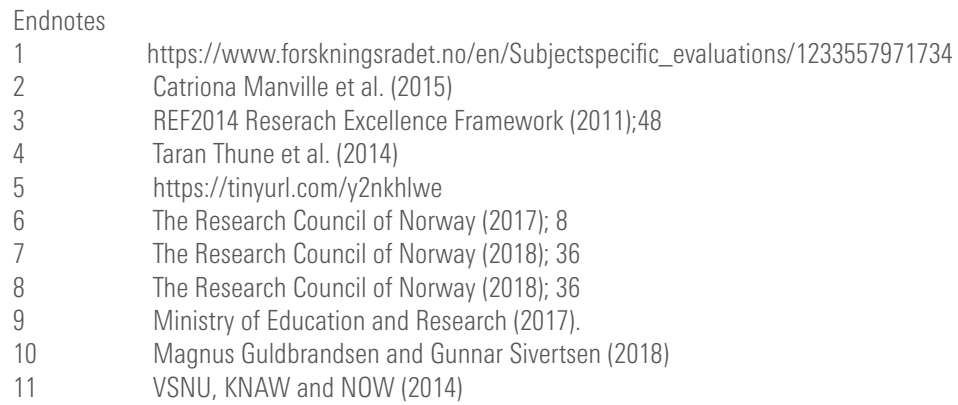

